\title{
INTOXICAÇÃO EXPERIMENTAL POR SEMENTES DE Crotalaria spectabilis (LEGUMINOSAE) EM SUÍNOS ${ }^{1}$
}

\author{
EXPERIMENTAL POISONING BY Crotalaria spectabilis \\ (LEGUMINOSAE) SEEDS IN PIGS
}

\author{
Márcia Bersane Araújo de Medeiros Torres² ${ }^{2}$ Mônica Weissmann Seabra Salles ${ }^{3}$ \\ Selwyn Arlington Headley ${ }^{4}$ Claudio Severo Lombardo de Barros ${ }^{5}$
}

RESUMO

Para estudar os efeitos da ingestão de sementes de Crotalaria spectabilis em suínos, 14 porcos foram divididos em 5 grupos; quatro (A-D) eram formados por 3 porcos e um (Grupo E) por dois porcos. Com exceção do grupo A, que serviu como controle, os porcos foram alimentados com ração contendo $0,3 e$ 0,5\% (Grupos B e C) e 1\% (Grupos D e E) de sementes moídas de C. spectabilis por 32 (Grupo D), 44 (Grupo E) e 120 (Grupo B e C) dias. Um suíno do Grupo D e 2 suínos do Grupo B, morreram 8,70 e 137 dias após o início do experimento; os restantes 11 suínos foram sacrificados em períodos que variaram entre 52 e 159 dias após o início do experimento. Dez dos 11 suínos tratados apresentaram perda do apetite e subdesenvolvimento. Outros sinais clínicos observados foram depressão, perda localizada de pêlos e distúrbios respiratórios. As principais lesões ocorreram no fígado. Em um caso eram agudas e consistiam principalmente de padrão lobular acentuado resultante de necrose centrolobular a massiva. Os outros 11 suínos apresentavam lesões hepáticas crônicas; o fígado estava firme, com cápsula espessada e aderências fibrosas entre os lobos e a estruturas adjacentes. Microscopicamente havia fibrose capsular, interlobar, interlobular $e$ intralobular; hepatomegalocitose e proliferação de ductos biliares. Bilestase foi observadada no fígado de dois suínos; num outro havia regeneração hepatocelular nodular. Os pulmões de 4 suí- nos estavam avermelhados e firmes. Histologicamente havia espessamento dos septos interalveolares e interlobulares por edema, fibrose e infiltração celular, e proliferação de pneumócitos tipo II. Alterações renais foram observadas apenas microscopicamente $e$ incluiam, principalmente, hepatomegalocitose das células epiteliais tubulares. Em 6 porcos observaram-se edemas cavitários (ascite, hidrotórax e hidropericárdio) de intensidade discreta a moderada. Alterações vasculares microscópicas consistindo de espessamento e hialinização da parede arterial foram observadas no pulmão de 5 suínos e no rim de 3. Quatro suínos tinham alterações sugestivas de encefalopatia hepática. Essas alterações não foram observadas nos suínos controles.

Palavras-chave: plantas tóxicas, Crotalaria spectabilis, Leguminosae, doenças de suínos, alcalóides pirrolizidínicos.

\section{SUMMARY}

To study the effects of the ingestion of Crotalaria spectabilis seeds, fourteen pigs were alloted in 5 groups. Four groups (A-D) consisted each of three pigs and the other (Group E) of two pigs. With the exception of those from Group A, which served as control, pigs were fed a ration containing 0.3 and $0.5 \%$ (groups

Parte da Dissertação de Mestrado apresentada pelo primeiro autor ao Curso de Pós-graduação em Medicina Veterinária, área de concentração em Patologia Veterinária da Universidade Federal de Santa Maria (UFSM), Santa Maria, RS. Trabalho financiado pelo CNPq.

${ }^{2}$ Médico Veterinário, Curso de Pós-graduação em Medicina Veterinária, área de Concentração em Patologia Veterinária, UFSM.

${ }^{3}$ Médico Veterinário, Professor Assistente do Departamento de Patologia (DP), UFSM.

${ }^{4}$ Aluno do Curso de Graduação em Medicina Veterinária da UFSM, bolsista PIBIC/CNPq.

${ }^{5}$ Médico Veterinário, Pesquisador 1A do CNPq (350938/91-1), Professor Titular, DP, Centro de Ciências da Saúde, UFSM, 97119-900 Santa Maria, RS. Autor para correspondência. 
$B$ and $C$ ) and 1\% (Groups $D$ and $E$ ) of ground seeds of $\boldsymbol{C}$. spectabilis for 32 (group D), 44 (group E), and 120 (groups B and $C$ ) days. One pig from Group $D$ and two pigs from Group $B$ died at 8, 70, and 137 days after the beginning of the experiment; the remaining 11 pigs were euthanatized at periods varying from 52 to 159 days from the beginning of the experiment. Ten of the 11 treated pigs had loss of apetite and poor growth rate. Depression, localized loss of hair, and respiratory distress were other observed clinical signs. Main pathological changes occurred in the liver. They were acute in one case and consisted of accentuation of the lobular pattern; due to centrilobular to massive hepatic necrosis. The remaining 11 treated pigs had chronic hepatic lesions, which included firmness, thickening of the capsule and fibrous adhesions between lobes and between hepatic capsule and adjacent structures. Histologically there was capsular, interlobar, interlobular, and intralobular fibrosis, hepatomegalocytosis and proliferation of bile ducts. In two case there was bilestasis and, in one pig, nodular regeneration was observed. The lungs of 4 pigs were reddened and firm. Histologically there was thickenning of interalveolar and interlobular septa by edema, fibrosis, cell infiltration and type II pneumocytes proliferation. The kidneys were grossly unremarkable. Histologically there was megalocytosis of the tubular epithelium and of the cells of the glomerular tuft. Hialinization of the glomerular tuft was also observed. Mild to moderate cavitary edema (ascites, hydrothorax and hydropericardium) was observed in 6 pigs. Microscopic vascular lesions consisting of arterial wall thickening and hialinization were seen in the lung of 5 pigs and in the kidney of 3 pigs. Four pigs had microscopic changes suggestive of hepatic encephalopathy. Those changes were not observed in the controls.

Key words: poisonous plants, Crotalaria spectabilis, Leguminosae, swine diseases, pirrolyzidine alkaloids.

\section{INTRODUÇÃO}

Plantas de várias famílias contêm alcalóides pirrolizídinicos (APs) e a intoxicação pela ingestão dessas plantas tem sido descrita em várias espécies domésticas (BULL $\boldsymbol{e t}$ al., 1968). Sabe-se que APs são principalmente hepatotóxicos (McLEAN, 1970). A espécie suína é considerada mais sensível, seguida, em ordem decrescente de susceptibilidade, por frangos, bovinos, eqüinos, ratos, camudongos, ovinos e caprinos (HOOPER, 1978)

O gênero Crotalaria (família Leguminosae) compreende cerca de 600 espécies (WILLIANS \& MOLYNEUX, 1987). Dessas, C. spectabilis é considerada como a mais tóxica (LORENZI, 1991); a toxicidade está relacionada ao AP monocrotalina presente nas folhas e sementes (WILLIANS \& MOLYNEUX, 1987). No Brasil cerca de 40 espécies de Crotalaria são conhecidas (TOKARNIA \& DÖBEREINER, 1983), mas há apenas um relato da toxicose espontânea por Crotalaria spp. no país (NOBRE $\boldsymbol{e} t$ al., 1994), embora existam evidências de que a intoxicação tenha ocorrido em eqüinos na Paraíba (NOBRE, 1994 - informe verbal) e em suínos no Rio Grande do Sul (DADOS NÃO PUBLICADOS).

Sinais clínicos da intoxicação em suínos consistem de redução no consumo de alimento e no ganho de peso (McGRATH \& DUNCAN, 1975), queda de pêlos pigmentados (EMMEL et al., 1935, GIBBONS, 1953, PECKHAM et al., 1974) e distúrbios respiratórios (PECKHAM et al., 1974). Os achados de necropsia podem ser agudos ou crônicos; os principais órgãos afetados são fígado, pulmões e rins (PECKHAM et al., 1974). Na microscopia as lesões hepáticas mais típicas são megalocitose, fibrose e proliferação de ductos biliares. Megalocitose é também observada no rim e pulmão (McLEAN, 1970, KELLY, 1993) associada à pneumonia intersticial.

O objetivo deste trabalho foi estudar os sinais clínicos, as lesões de necropsia e alterações histológicas relacionadas à ingestão de sementes de $\boldsymbol{C}$. spectabilis em suínos.

\section{MATERIAIS E MÉTODOS}

Foram utilizados 14 suínos mantidos em baias individuais. Doze eram cruza Landrace $\mathrm{x}$ Large White, tinham 45 dias de idade e pesavam $12 \mathrm{~kg}$. Esses animais foram divididos em 4 grupos de 3 suínos (Grupos A-D). Os animais do grupo A eram fêmeas e serviam como controle. Um outro grupo (E), era constituído de duas fêmeas, Duroc x Pietrain, com 180 dias de idade e $28 \mathrm{~kg}$. Esse grupo visava incluir fêmeas de pele escura. A ração basal era produzida pelo Setor de Zootecnia da UFSM com composição adequada à fase de crescimento dos suínos..

Sementes maduras de $\boldsymbol{C}$. spectabilis foram secadas em estufa à 55?C por 72 horas, trituradas e misturadas à ração basal em diferentes concentrações. $\mathrm{O}$ grupo controle recebia somente a ração basal. Os grupos B, C, D e E recebiam a ração basal adicionada respectivamente de $0,3 \%, 0,5 \%, 1,0 \%$ e $1,0 \%$ de sementes de C. spectabilis. Os suínos receberam a ração com sementes por 32 (grupo D), 44 (grupo E) e 120 dias (D e E). Antes de serem misturadas à ração, amostras das sementes foram submetidas a exame para pesquisa de micotoxinas.

Todos os suínos que não morreram em consequiência da intoxicação foram sacrificados; todos foram necropsiados. Fragmentos de diversos órgãos foram fixados em formol a $10 \%$ e processados rotineiramente para histopatologia. Cortes selecionados do fígado foram corados pelo Tricrômico de Masson e Sudan III. 


\section{RESULTADOS}

Três suínos tiveram morte espontânea e os demais foram sacrificados em vários tempos após início do experimento (Tabela 1). Perda de apetite, em graus variáveis, foi observada em todos os suínos, exceto no suíno 313. Apesar dos animais não terem sido pesados após o início do experimento, pôde-se observar que, exceto nos do grupo controle, não houve ganho de peso normal. Queda de pêlos foi observada em todos os suínos dos grupos BE, no dorso e região periocular; nos animais Duroc $x$ Pietrain a alopecia era restrita as áreas pigmentadas.

Tabela I - Intoxicaça experimental por sementes de Crotafaria spectabilis (Leguminosuc) em sulnos. Destecho do cxperimento por animal e grupo c periodo, em dias, da morte ou sacrificio aposs o inleto do experimente.

\begin{tabular}{|c|c|c|c|}
\hline Suíne & Grupo & $\begin{array}{l}\text { Morte espontănea } \\
\text { (M)ou sacrificio(S) }\end{array}$ & $\begin{array}{l}\text { Morte após } \\
\text { experimento } \\
\text { (eni dias) }\end{array}$ \\
\hline 19 & A & s & 96 \\
\hline 108 & $A$ & $\$$ & 159 \\
\hline 109 & $A$ & $S$ & 159 \\
\hline 393 & B & $M$ & 70 \\
\hline 75 & B & $M$ & 137 \\
\hline 101 & $B$ & $\$$ & 155 \\
\hline 387 & $c$ & S & 52 \\
\hline 16 & $c$ & $\mathrm{~S}$ & 95 \\
\hline 104 & c & $\mathrm{S}$ & 157 \\
\hline 313 & D & $M$ & 8 \\
\hline 54 & D & S & 114 \\
\hline 102 & D & $S$ & 156 \\
\hline 189 & $\mathrm{E}$ & $S$ & 61 \\
\hline 229 & $\mathrm{E}$ & $S$ & 99 \\
\hline
\end{tabular}

Tosse, temperatura de 40 ? e decúbito permanente foram observados no suíno 16 a partir do $78^{\circ}$ dia do experimento; a partir do $132^{\circ}$ dia o suíno 75 apresentou respiração abdominal, taquipnéia e anorexia; esses sinais persistiram até a morte do animal.

Na necropsia dos 11 suínos que receberam as sementes de $\boldsymbol{C}$. spectabilis, observou-se de modo geral, má condição corporal. A cavidade abdominal de três suínos continha excesso de líquido, com aspecto variando de citrino no suíno 75 , de coloração marrom-escura e com fibrina e conteúdo gástrico proveniente de úlcera gástrica perfurada no suíno 387 e sanguinolento no suíno 313. O suíno 75 apresentava $200 \mathrm{ml}$ de líquido claro com fibrina na cavidade torácica.

Alterações pulmonares como aumento da consistência foram evidentes em 4 suínos $(54,75$, $102,229)$, essa lesão era acentuada no suíno 75 , no qual o pulmão estava avermelhado, a superfície de corte tinha septos interlobulares evidentes e havia áreas esbranquiçadas intercaladas por áreas avermelhadas. A mucosa da região gastresofágica de 3 suínos $(387,75,101)$ estava ulcerada; no suíno 387 havia perfuração da úlcera. O suíno 313 tinha alterações hepáticas agudas; o fígado tinha coloração avermelhada, havia filamentos de fibrina na superfície capsular e acentuação do padrão lobular; os demais suínos tinham lesões crônicas caracterizadas por aumento da consistência, espessamento da cápsula de Glisson, aderências fibrosas entre os lobos, diminuição do tamanho do órgão e alterações da coloração que variavam de amarelo-alaranjado a moteado com áreas marromclaras e áreas vermelhas; o fígado do suíno 102 tinha nódulos aleatórios amarelados de $0,3 \mathrm{~cm}$ de diâmetro no parênquima. Edema da vesícula biliar foi observado em 3 suínos.

Microscopicamente a lesão hepática aguda era caracterizada por necrose e hemorragia centrolobulares; a lesão crônica caracterizava-se por hepatomegalocitose, proliferação ductal e fibrose (Figura1), necrose hepatocelular individual ou de grupos celulares e infiltrado mononuclear ou neutrocitário. Bilestase foi vista nos suínos 101 e 229 e nódulos de regeneração no suíno 102.

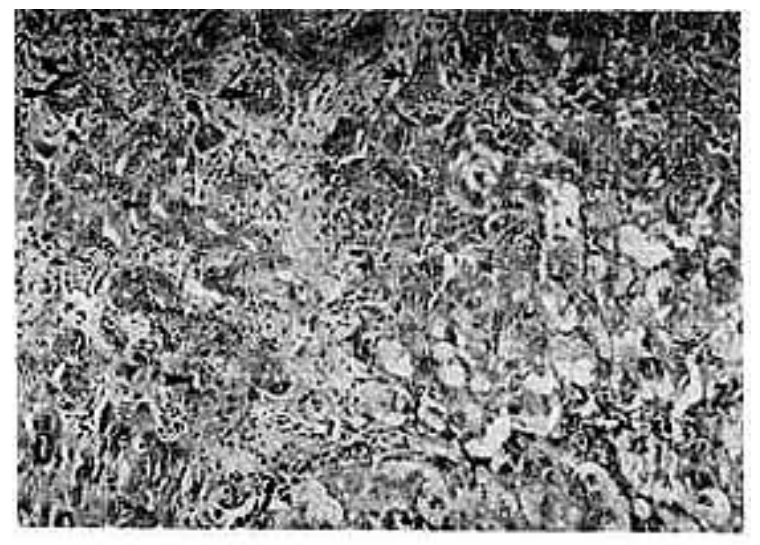

Figufa 1 - Intoxicaçăo experimental por sementes de Crovaloria spectabilis (Leguminosac) em suinos. Sulmo 102, Gngo D. Figado. Acentuada proliferaçalo de ductos biliares (cabeças de seta) $\mathrm{cm}$ meio a fibrose. Obj. 10. H.E. 
As hemorragias observadas em casos agudos, estão, provavelmente, relacionados à diminuição na síntese dos fatores da coagulação por insuficiência hepática ou ao efeito tóxico direto sobre as células endoteliais (ALLEN, 1963). Edemas cavitários observados foram semelhantes ao que é descrito em suínos (EMMEL, 1935, PECKHAM et al., 1974, SOUZA, 1996) cavalos (GIBBONS et al., 1953) e aves (FIGUEREDO $\boldsymbol{e t}$ al., 1987), intoxicados por $\boldsymbol{C}$. spectabilis. Os edemas associados à intoxicação por Crotalaria spp. têm sido atribuídos à diminuição da síntese protéica pelo fígado lesado (PETTERSON \& CULVENOR, 1983, SOUZA, 1996) ou à hipertensão portal por lesão hepática crônica (PETTERSON \& CULVENOR, 1983, KELLY, 1993) ou ambos (PETTERSON \& CULVENOR, 1983).

As alterações hepáticas vistas na intoxicação crônica são clássicas da intoxicação por APs (BULL et al., 1968, McLEAN, 1970). Lesões hepáticas crônicas devem ser diferenciadas principalmente das causadas por aflatoxinas (KELLY, 1993). O efeito mais característico dos metabólitos dos APs sobre o fígado é a megalocitose que ocorre em função de um efeito antimitótico, sem inibição da síntese de DNA; a síntese continuada de DNA, combinada com a inibição mitótica, resulta no aumento da célula (McLEAN, 1970).

A megalocitose das células epiteliais dos túbulos renais foi observada em 10 suínos e é descrita na intoxicação por outras espécies de Crotalaria (McGRATH \& DUNCAN, 1975, HOOPER \& SCANLAN, 1977, PETERSON \& CULVENOR, 1983) em suínos, mas raramente ocorre nesta toxicose em outras espécies. A maior freqüência de megalocitose nas células renais em suínos pode ser atribuída a uma maior excreção do metabólito pirrólico, ingerido como AP, na urina nessa espécie ou ao fato dos túbulos renais dos suínos concentrarem o metabólito mais eficientemente (PETERSON \& CULVENOR, 1983).

O mecanismo da lesão glomerular em suínos na intoxicação por APs não é esclarecido. Células mesangiais são acentuadamente sensíveis a estímulos nocivos e respondem à injúria com aumento na deposição de matriz mesangial (NAGASE et al., 1971). APs são hidrolisados no fígado a metabólitos pirrólicos (McLEAN, 1970) e hidrólises subseqüentes produzem álcoois pirrólicos que são excretados na urina (BULL et al., 1968). É possível que essa seja a explicação para o aumento de material eosinfílico no tufo glomerular e que a injúria, nesse caso, seja a passagem de metabólitos de APs pelo filtrado glomerular. Que metabólitos de APs sejam irritantes para o rim em suínos já foi sugerido anteriormente (BULL et al., 1968).

Lesões pulmonares na intoxicação por Crotalaria spp. são descritas como de dois tipos: lesões vasculares (HURLEY \& JAGO, 1976) e reação celular do interstício (BARNES,1964), embora seja sugerido que as lesões vasculares são apenas o estágio inical das lesões do parênquima pulmonar (McGRATH \& DUNCAN, 1975). Reações do interstício caracterizadas por pneumonia intersticial, semelhante ao observado nesse experimento são descritas na intoxicação por Crotalaria spp. em suínos (PECKHAM et al., 1974, HOOPER \& SCANLAN, 1977), cavalos (NOBRE et al., 1994), bovinos (LEMOS, 1996 - informe verbal) e em cavalos intoxicados por Eupatorium spp. (O’SULLIVAN, 1979) e suínos intoxicados por Senecio jacobaea (HARDING et al., 1964).

Alterações degenerativas e proliferativas em capilares, arteríolas e artérias pequenas e médias foram observadas no pulmão e rim dos suínos deste experimento. Lesões semelhantes têm sido descritas na intoxicação por Crotalaria spp. em suínos (PECKHAM et al., 1974) e em ratos ( GHODSI \& WILL, 1981).

As lesões sugestivas de encefalopatia hepática observadas nesse experimento estão relacionadas à insuficiência hepática e conseqüente hiperamonemia (HOOPER, 1978). Não se observaram sinais clínicos claramente associados a essa lesão.

\section{INFORME VERBAL}

LEMOS, Ricardo Antônio Amaral de. 1996. Departamento de Medicina Veterinária. Universidade Federal de Mato Grosso do Sul. 79070-900, Campo Grande, MS.

NOBRE, Verônica Medeiros da Trindade.1994. Departamento de Medicina Veterinária, Universidade Federal da Paraíba, Campus VII, 58700-000, Patos, PB.

\section{REFERÊNCIAS BIBLIOGRÁFICAS}

ALLEN, J.R. Crotalaria spectabilis toxicity studies in turkeys. Avian Dis., v. 7, n. 3, p. 318-324, 1963.

BARNES, J.M., MAGEE, P.N., SCHOENTAL, R. Lesions in the lungs and livers of rats poisoned with the pyrrolizidine alkaloid fulvine and its N-oxide. J Path Bact, v. 88, p. 521-531, 1964.

BULL, L.B., CULVENOR, C.C.J., DICK, A.T. The pyrrolizidine alkaloids. Their chemistry, pathogenicity and other biological properties. North-Holland Publ., 1968. 293 p.

EMMEL, M.W., SANDERS, D.A., HENLEY, W.W. Crotalaria spectabilis Roth seed poisoning in swine. J Am Vet Med Assoc, v. 86, n. 1, p. $43-45,1935$. 
EMMEL, M.W. Livestock poisoning by Crotalaria spectabilis. Vet Med, p. 255-257, 1948.

FIGUEREDO, M.L.F., RODRIGUEZ, J., ALFONSO, H.A. Patomorfologia de la intoxicación experimental aguda por Crotalaria retusa y $C$. spectabilis en pollos. Rvta Cub Cien, v. 18, n. 1 e 2 , p. 63-71, 1987.

GHODSI, F., WILL, J.A. Changes in pulmonary structure and function induced by monocrotaline intoxication. Am Phy Soc, p. $149-155,1981$.

GIBBONS, W.J., DURR, E.H., COX, S.A. An outbreak of cirrhosis of the liver in horses. North Amer Vet, v. 34, p. 556-558, 1953.

HARDING, J.D.J., LEWIS, G., DONE, J.T., ALLCROFT, R. Experimental poisoning by Senecio jacobae in pigs. Path Vet, v. 1, p. 204-220, 1964.

HOOPER, P.T. Pyrrolizidine alkaloid poisoning - Pathology with particular reference to differences in animal and plant species. In: KEELER, R., VAN KAMPEN, K.R., JAMES, L.F. Effects of poisonous plants on livestock. New York: Academic Press. 1978. cap. s/n, p. 600.

HOOPER, P.T., SCANLAN, W.A. Crotalaria retusa poisoning of pigs and poultry. Aust Vet J, v. 53, p. 109-114, 1977.

HURLEY, J.V., JAGO, M.V. Delayed and prolonged vascular leakage in inflammation: the effects of dehydromonocrotaline on blood vessels in the rat Cremaster. Pathology, v. 8, p. 7-20, 1976.

KELLY, W.R. The liver and biliar system. In: JUBB, K.V.F., KENNEDY, P.C., PALMER, N. Pathology of domestic animals. 4. ed. San Diego: Academic Press, 1993. v. 2, cap. 2, p. 319-406.

LORENZI, H. Plantas daninhas do Brasil. 2. ed. Nova Odessa: Editora Plantarum, 1991, 440 p.

McGRATH, J.P.M., DUNCAN, J.R. Crotalaria spectabilis toxicity in swine: characterization of the renal glomerular lesions. $\mathbf{J}$ Comp Path, v. 85, p. 185-194, 1975.

McLEAN, E.K. The toxic actions of pyrrolizidine (Senecio) alkaloids. Pharmacological reviews, v. 22, n. 4, p. 429-483, 1970.
NAGASE, M., IGARASHI, S., YOSHITOSHI, Y. Studies on the glomerular basement membranes. I.3H-proline incorporation into glomeruli of uranium injected rats, studied on isolated glomerular basement membranes and autoradiogram. Jap J Exp Med, v. 41, p. 547-555, 1971.

NOBRE, D., DAGLI, M.L.Z., HARAGUCHI, M. Crotalaria juncea intoxication in horses. Vet Hum Toxicol, v. 36, n. 5, p. 445448, 1994.

O'SULIVAN, B.M. Crofton weed (Eupatorium adenophorum) toxicity in horses. Aust Vet J, v. 55, p. 19-21, 1979.

PECKHAM, J.C., SANGSTER, L.T., JONES, Jr.O.H. Crotalaria spectabilis poisoning in swine. J Am Vet Med Assoc, v. 165, n. 7, p. 633-638, 1974.

PETERSON, J.E., CULVENOR, C.C.J. Hepatotoxic pyrrolizidine alkaloids. In: KEELER, R.F., TU, A.T. Handbook of natural toxins. New York: Marcel Dekker, 1983. v. 1, cap. 19, p. 637671 .

ROSS, A. Effects of feeding diets containing Crotalaria retusa L. seed to growing pigs. J Agric Sci, v. 89, p. 101-105, 1977.

ROTH, R.A., DOTZLAL, L.A., BARANYI, B., KUO, C.H., HOOK, J.B. Effect of monocrotaline ingestion on liver, kidney and lungs of rats. Toxicol and Appl. Pharmacol, v. 60, p. 193-203, 1981.

SIPPEL, W.L. Crotalaria poisoning in livestock and poultry. Ann N Y Acad Sci, v. 111, p. 562-570, 1964.

SOUZA, A.C. Intoxicação experimental de suínos com sementes de Crotalaria spectabilis (Fabaceae). Jaboticabal: Faculdade de Ciências Agrárias e Veterinárias do Campus de Jaboticabal- UNESP, 1996. 104 p. Dissertação (Mestrado em Medicina Veterinária - Área de concentração em Patologia Animal). Curso de Pós-graduação em Medicina Veterinária. Faculdade de Ciências Agrárias e Veterinárias do Campus de Jaboticabal- UNESP, 1996.

C.H., DÖBEREINER, J. Intoxicação experimental por Crotalaria anagyroides (Leg. Papilionoide) em bovinos. Pesq Vet Bras, v. 3, n. 4, p. $115-123,1983$.

WILLIANS, M.C., MOLYNEUX, R.J. Occurrence, concentration, and toxicity of pyrrolizidine alkaloids in crotalaria seeds. Weed Sci, v. 35, n. 4, p. 476-481, 1987. 